\title{
Las ciclovías, la movilización de las personas y su salud
}

\author{
Bicycle lanes, the mobilization of people and their health
}

\author{
Mitzi Lourdes del Carmen Linares-Vizcarra \\ Universidad Privada de Tacna \\ Tacna, Perú \\ mitlinaresv@upt.pe \\ https://orcid.org/0000-0003-0471-8856 \\ — [Presentado: 10/05/2021, Aceptado: 08/07/2021]
}

\begin{abstract}
RESUMEN
Uno de los problemas en Perú es el caótico transporte automotor, así como el incremento de las migraciones, esto conlleva al crecimiento del parque automotor, no solo en la capital, sino en las capitales de departamento. De ahí que el parque automotor esté al borde del colapso, ya que es muy antiguo, y es la causa de los problemas de espacio en la ciudad, la contaminación, la vida sedentaria y el tiempo que los usuarios disponen para trasladarse. Tacna no escapa de esta realidad, pero el problema es más notorio en la capital de Perú. Ante ello, las ciclovías surgen como una solución, debido a que contribuyen al desarrollo articulado de la infraestructura urbana, que debiera estar acorde con las necesidades sociales y avances tecnológicos. Se utilizó el método deductivo, ya que se observó la realidad y se trata de dar una solución alternativa al incremento desmedido del parque automotor. Entre los resultados inmediatos se verá el incremento del uso de las bicicletas y la mejora de la calidad de vida, reflejada en la salud de la población. Se concluye que es importante que el gobierno destine un presupuesto para el crecimiento integral de las ciclovías, lo que conlleva al surgimiento y crecimiento de emprendimientos, así como a la mejora de la salud.
\end{abstract}

Palabras clave: Ciclovías, parque automotor, factores positivos, salud.

\begin{abstract}
One of the problems in Peru is the chaotic automobile transport, as well as the increase in migrations, this leads to the growth of the automobile fleet, not only in the capital, but also in the departmental capitals. Hence, the fleet is on the verge of collapse, since it is very old, and is the cause of space problems in the city, pollution, sedentary life and the time that users must move. Tacna does not escape this reality, but the problem is more noticeable in the capital of Peru. Given this, bicycle lanes emerge as a solution, because they contribute to the articulated development of urban infrastructure, which should be in line with social needs and technological advances. The deductive method was used, since reality was observed and it is about providing an alternative solution to the excessive increase in the number of vehicles. Among the immediate results will be the increase in the use of bicycles and the improvement of the quality of life, reflected in the health of the population. It is concluded that it is important for the government to allocate a budget for the comprehensive growth of bicycle lanes, which leads to the emergence and growth of enterprises, as well as the improvement of health.
\end{abstract}

Keywords: Bicycle lanes, vehicle fleet, positive factors, health. 


\section{INTRODUCCIÓN}

En el Perú, debido a los beneficios que se obtienen en algunas zonas, como las de tratamiento especial en los que se importan vehículos, y lo fácil que es para utilizarlos como herramientas de trabajo, es que el parque automotor se ha visto rebasado, en la medida que no solo circulan vehículos nuevos, sino que más de la mitad de ellos, ya han sobrepasado los cuatro años de vida útil, lo cual hace que tengamos un parque automotor que linda con la obsolescencia, además de una fuerte contaminación ambiental, además de disminuir las posibilidades de que se incrementen las ciclovías no solo en Lima, sino que también en las provincias, por tanto, es procedente preguntarse: ¿Las ciclovías contribuyen a la movilización de las personas y su salud? ¿Es factible que se habiliten ciclovías en Tacna?

En el Perú, se están habilitando espacios para la circulación de ciclos ${ }^{1}$; y esta actividad se ha visto impulsada a partir del Decreto Supremo N. ${ }^{\circ}$ 005-2020-MTC, que modifica los requisitos mínimos de calidad para la importación de vehículos usados establecidos en el Decreto Legislativo N. ${ }^{\circ} 843$, indica, en su Artículo 1.- ... a) Que tengan una antigüedad no mayor de dos años. La antigüedad de los vehículos se cuenta a partir del año modelo. Queda prohibida la importación de vehículos usados con motor de encendido por compresión (diésel) de las categorías L, M y N, a excepción de las subcategorías M3 y N3 (Perú, 2020), por lo que se espera que el parque automotor disminuya ostensiblemente y sea proporcional a la contaminación ambiental.

Asimismo, se ha publicado el Decreto Supremo, que aprueba el Reglamento de la Ley N. 30936, referida a la Ley que promueve y regula el uso de la bicicleta como medio de transporte sostenible, que modifica el Reglamento Nacional de Tránsito, aprobado por Decreto Supremo N. ${ }^{\circ}$ 033-2001-MTC y el Reglamento Nacional de Gestión de Infraestructura Vial, aprobado por Decreto Supremo N. ${ }^{\circ}$ 034-2008MTC, aprobándose el Reglamento de la Ley 30936 mediante el Decreto Supremo N. ${ }^{\circ}$ 012-2020-MTC, en el que se determina que el financiamiento será de cargo al presupuesto institucional aprobado de los pliegos involucrados, sin demandar recursos adicionales al Tesoro Público. Asimismo, en su primera Disposición Complementaria Final, se dispone que "Las papeletas que se impongan por la comisión de las infracciones establecidas en el numeral III. CONDUCTORES/AS DE BICICLETA U OTRO CICLO DEL ANEXO "CUADRO DE TIPIFICACIÓN, SANCIONES Y MEDIDAS PREVENTIVAS APLICABLES A LAS INFRACCIONES AL TRÁNSITO TERRESTRE” del Reglamento Nacional de Tránsito, aprobado por Decreto Supremo No 033-2001-MTC y modificatorias, tienen carácter educativo durante un (1) año contado desde la vigencia del presente Decreto Supremo, plazo en el cual el Ministerio de Transportes y Comunicaciones evalúa las conductas infractoras y la implementación del presente Decreto Supremo." (Perú, Decreto Supremo 012-2020-MTC, 2020). Estas modificatorias a la legislación, harán que el trámite para la implementación de ciclovías sea sencillo, menos burocrático, además de atender a las necesidades de la población, originar ideas de emprendimientos, abrir un interesante mercado a expandir, así como la promoción del uso de bicicletas.

Esto conlleva al empuje que se les dará a los técnicos y profesionales que se dedican al mantenimiento de los ciclos. De igual manera, en el Manual para ciclistas del Perú, ya se determinan las reglas y recomendaciones para el uso de la bicicleta y otros ciclos, de forma que respeten las señales de tránsito, lo que conlleva a su respeto por terceros.

1 Todos los vehículos accionados por el trabajo muscular y que no forman parte de los vehículos automotores ni de los vehículos eléctricos. (Ministerio de Transportes y Comunicaciones, 2020) 
En Tacna, se está viendo la posibilidad de habilitar ciclovías, y esto es consecuencia del tiempo en que vivimos, no solo por la pandemia, sino porque existe una tendencia de vida sana, y por ello, el movilizarse en bicicleta es una buena opción para llegar al centro laboral, por ejemplo, se ha demostrado científicamente, que el ejercicio produce endorfinas que hacen que el ser humano sea más ágil, se encuentre con mayor disposición a cumplir con su trabajo y elimine el mal humor, además de que para las empresas sería una acción de responsabilidad social.

En Latinoamérica, Lima Metropolitana es una de las áreas metropolitanas más contaminadas, con el $80 \%$ de polución, que es consecuencia de su parque automotor obsoleto, conforme lo ha indicado la Organización Mundial de la Salud, lo que va aunado a la pésima calidad de los combustibles (Vilma Tapia, 2018).

En la capital de Perú se concentra la mayor cantidad de la población, precisamente por la migración, esto hace que las ofertas laborales escaseen y la demanda se incremente, por tanto, al no existir un trabajo adecuado y constante en el tiempo, los migrantes, en su mayoría, han optado por dedicarse a cualquier actividad que les reditúe un ingreso económico, una de ellas son las empresas de locomoción pública, pues es una fuente de dinero para poder proveer a sus familias, sin aportar en lo mínimo al erario nacional, pues al ser informales en su mayoría no tributan, lo que es consecuencia de una falta de conciencia tributaria.

La tendencia nacional, además de una cultura de paz, es la de la vida sana, y por ello, varios distritos de la capital del Perú tienen ciclovías, aunque aún no son suficientes; sin embargo, es importante, indicar que se han dado normas legales a efectos de fomentar la construcción de ciclovías, o en su caso adecuar espacios para ellas.

Ahora, desde el pasado año, se promueve el uso de la bicicleta para movilizarse, casi como una forma de traslado obligatorio, en la medida que por las cuarentenas, se ha prohibido el uso de los vehículos particulares, lo que ostensiblemente ha hecho que mejore el hábitat, disminuya el smog, la naturaleza recobre su espacio, por lo menos en la primera cuarentena, en la que se podían ver a los animales recobrando su hábitat, y ver el mar relativamente limpio, lo que hacía desear que fuera una política gubernamental, que se hiciera lo mismo en cada gobierno. Sin embargo, es imposible, ya que por una parte se lograba recuperar esa naturaleza, pero por otro lado, la población de escasos recursos no podía sobrevivir tanto tiempo sin salir a trabajar, por lo que el negocio de los delivery creció, pero igual que ellos, crecieron los amantes del transporte en bicicleta, al no tener automotores en circulación, se hacía más sencillo transportarse en bicicleta, formar un hábito y obtener una mejor salud, con tiempo para confraternizar entre los colaboradores de las empresas que requerían trabajo presencial.

Es importante indicar que, gracias a las modificaciones legales, el financiamiento para la construcción de ciclovías se encuentra garantizado, así que dependerá de la gestión de los gobiernos descentralizados la procuración de espacios adecuados para el transporte en bicicleta.

\section{MATERIAL Y MÉTODOS}

Para este trabajo se ha consultado a varios autores como: Alicia Castro que es profesora de Educación Secundaria en la especialidad de Física y Matemática, cuenta con el grado de Magíster en Psicología 
con mención en Problemas de Aprendizaje y es docente de la Facultad de Educación de la Universidad Peruana Cayetano Heredia. La Municipalidad de Lima, que es el órgano responsable de la administración municipal, la cual debe promover el crecimiento urbano ordenado, por tanto, es responsable de planear, conducir, establecer, ejecutar, evaluar y supervisar las acciones y actividades que desarrolle. (Municipalidad de Lima, 2014: 1). La Defensoría del Pueblo, cuyo objetivo primordial es el atender las quejas en todo el país, es el ente ante el que se puede efectuar consultas, pedidos, ya que resguarda los derechos, y evita su vulneración ante instancias estatales (Defensoría del Pueblo 2014: 1). El Instituto Nacional de Estadística e Informática (INEI) que se encarga de "Producir y difundir información estadística oficial que el país necesita con calidad, oportunidad y cobertura requerida" (INEI 2014: 1). Así también, al escritor Oscar Ponce de León que es profesor de Microeconomía y Pregrado en la Universidad del Pacífico y es también director ejecutivo en ProInversión. Diario El Peruano, en el que se publicaron los dispositivos que regulan las importaciones de vehículos con antigüedad no mayor a dos años, así como las facilidades para que se construyan ciclovías a nivel nacional.

Entre otras referencias, debemos preponderar al Manual para Ciclistas del Perú, que contiene las reglas y recomendaciones para el uso de bicicletas y otros ciclos, en el que se indican, incluso, las partes de la bicicleta, la mecánica básica, las reglas de circulación, el uso de las vías públicas, el equipamiento de la bicicleta u otros ciclos y del ciclista.

Se ha utilizado la técnica de la observación, se ha utilizado bibliografía, webgrafía y estadística que describen experiencias y plantean propuestas viables que coinciden con lo que se proyecta, que no es más que plantar conciencia de la importancia de la salud y lo perjudicial que es continuar con el aumento del parque automotor desmedidamente sin que los gobiernos locales hagan algo para mejorar la calidad de vida las personas y disminuir la contaminación del medio ambiente.

En este siglo, y en esta coyuntura, se hace necesaria la inversión privada y la inversión de los gobiernos locales en el desarrollo de la infraestructura de ciclovías y del incremento de estos vehículos, así como los emprendimientos destinados a la transacción de bicicletas, sus accesorios y equipos de seguridad para los que la utilicen, sin dejar de lado las facilidades que deberán proporcionar los empleadores con el objetivo de asegurar los vehículos menores y fomentar el transporte de sus colaboradores.

La empresa tendrá un beneficio en el rendimiento de sus colaboradores, y el clima organizacional, como el comportamiento dentro de la organización, mejorará ostensiblemente.

Conforme a lo ya mencionado, debemos reiterar la importancia de tener una vida sana y un mejor medio ambiente, por lo que surgen las preguntas: ¿Qué hacer? ¿Cómo hacerlo? ¿Cuándo hacerlo?, el por qué, ya se respondió.

Por tanto, debemos indicar la actuación esencial e importante que cumplen los municipios al resguardar el desarrollo urbanístico adecuado para hacer eficiente los espacios, al obtener resultados óptimos, que es el objetivo, ya sea con el uso de las ciclovías como también con la mejora de la salud de cada una de las personas que conforman diversos distritos.

Se tiene conocimiento que la actividad física deportiva es menor al $12 \%$, por lo que el promedio en Perú es que uno de cada dos peruanos no practica ningún deporte (Lao, 2021), lo que se hace crítico en la zona urbana a consecuencia del parque automotor y la rapidez con la que se vive, con el tiempo siempre en contra y las personas estresadas, sin saber cómo manejar el estrés y que luego no solo es llevado al trabajo sino al hogar, lo que perjudica ambos ámbitos en los que debe desarrollarse. Es aquí 
donde notamos que los limeños no están acostumbrados a realizar actividades físicas básicas o las que se pueden encontrar en las calles como las ciclovías que son una herramienta más accesible que no tiene ningún tipo de inversión y sin embargo las personas no se dan tiempo para dedicarle un buen uso.

Incluso, las ciclovías se han vuelto un ejemplo de sistema en muchos países, como Bogotá que tiene "La ciclovía más concurrida, la más extensa y la segunda más antigua del mundo" (Municipalidad de Lima 2013: 4). Es por esta razón que se debe utilizar este medio de transporte que no es de importancia para algunas personas, ni para el Estado, pero que trae bastante desarrollo y mejora en el sistema de transporte, debido a la versatilidad de las ciclovías y mejor manejo del tiempo. Además, "Permiten un espacio para hacer ejercicio a las personas que usualmente no hacen deporte." (Municipalidad de Lima 2013: 6), ya que no solo son utilizadas para el uso de los ciclistas y deportistas que utilicen esta rama del sistema de transporte para beneficio propio, sino que cualquier persona puede utilizarla de manera personal, para su beneficio corporal y mental.

En consecuencia, es necesario soluciones inmediatas para incentivar a las personas a utilizar las ciclovías, ya que "Es una calle, avenida o cualquier vía que es liberada de vehículos con motor por un tiempo (varias horas de algunos días) para que sea transitada solamente a pie o por vehículos sin motor" (Municipalidad de Lima 2013: 2). Además, el Ministerio de Salud afirma que para el año 2025, las personas sufrirán, en gran porcentaje, enfermedades cardiovasculares y neoplasias, debido a la falta de la cultura de "vida sana" que es consecuencia de "la cultura del deporte" (Mogrovejo Jaramillo, 2016). Esto se verá reflejado por la falta de actividad física que las personas podemos realizar en un determinado tiempo, por ello, lo recomendable es utilizar por lo menos las ciclovías que brinda, en determinados distritos, la ciudad de Lima.

Estas ciclovías "No impiden la entrada y la salida de vecinos en vehículos - No interrumpe totalmente el transporte motorizado" (Municipalidad de Lima 2013: 7), es por tal razón que el sistema de ciclovías tiene un buen rendimiento a la hora de utilizarlas.

Podemos destacar que existen muchos motivos por el cual el desarrollo de las ciclovías es de poco uso en Lima Metropolitana. Sin duda, la mayor dificultad es que las diferentes municipalidades no tienen un plan de mejora para mantener e implementar vías alternas de uso exclusivo para los ciclistas. A su vez, se puede afirmar que será difícil adquirir una eficaz infraestructura de asociaciones públicas-privadas para la inversión en ciclovías. Asimismo, el sector privado tampoco hace aporte a la protección ni implementación de estas vías. Es por eso por lo que, el Estado se ha visto obligado de implementar las normas indicadas, modificándolas de forma que sea aplicable a la realidad y a la coyuntura, esto facilita la inversión de los gobiernos regionales y locales, así como la asociación con privados para que aporten un financiamiento que permita el mantenimiento de estas vías.

Los arbitrios que son un impuesto para la mejora y el mantenimiento de las vías públicas no cubren el mantenimiento y la preservación de las ciclovías, debido a que las municipalidades de capital de departamentos y los demás distritos le dan más importancia a los parques, pistas y veredas. Este es el principal factor por el cual no se puede dar la implementación de estas vías de transporte alterno.

Es de suma importancia dar un vistazo a las cifras de accidentes de tránsito, ya que, al hacer referencia a vehículos mayores, involucra a automóviles, station wagon, camionetas, camiones, remolcador, furgoneta y ómnibus; y cuando se hace referencia a vehículos menores están comprendidos las motos, motocarros, bicicletas y triciclos, los cuales son de producción nacional o importada. A continuación, se detallan los siguientes datos según el INEI (tablas 1 y 2). 


\section{Tabla 1}

Perú: Accidentes de tránsito por cada 10000 vehículos del parque automotor a nivel nacional

\begin{tabular}{lccc}
\hline Nacional & $\begin{array}{c}\text { N. }^{\circ} \text { de accidentes de } \\
\text { tránsito }\end{array}$ & $\begin{array}{c}\text { N. }^{\circ} \text { de vehículos del } \\
\text { parque automotor 1/ }\end{array}$ & $\begin{array}{c}\text { Accidentes de tránsito por } \\
\text { cada 10 000 vehículos }\end{array}$ \\
\hline Total & 99170 & 2826909 & 351 \\
\hline
\end{tabular}

Fuente: Información proporcionada por la Superintendencia Nacional de Registros Públicos (SUNARP)

Tabla 2

Perú: Vehículos menores involucrados en accidentes de tránsito, según departamento, 2009

\begin{tabular}{|c|c|c|c|}
\hline Departamento & N. ${ }^{\circ}$ de vehículos & $\begin{array}{c}\text { N. }{ }^{\circ} \text { de vehículos } \\
\text { menores }\end{array}$ & $\%$ de vehículos menores \\
\hline Total & 159214 & 31279 & 19.6 \\
\hline Amazonas & 491 & 167 & 34.1 \\
\hline Ancash & 4455 & 740 & 16.6 \\
\hline Apurímac & 466 & 46 & 9.9 \\
\hline Arequipa & 7795 & 534 & 6.9 \\
\hline Ayacucho & 2582 & 509 & 19.7 \\
\hline Cajamarca & 4019 & 1050 & 26.1 \\
\hline Callao & 4890 & 773 & 15.8 \\
\hline Cusco & 5704 & 686 & 12 \\
\hline Huancavelica & 331 & 37 & 11.2 \\
\hline Huánuco & 1502 & 630 & 42 \\
\hline Ica & 2942 & 884 & 30 \\
\hline Junín & 4610 & 631 & 13.7 \\
\hline La Libertad & 6057 & 1354 & 22.3 \\
\hline Lambayeque & 4293 & 1764 & 41.1 \\
\hline Lima & 90331 & 12841 & 14.2 \\
\hline Loreto & 1440 & 1182 & 82 \\
\hline Madre de Dios & 617 & 457 & 74 \\
\hline Moquegua & 1187 & 89 & 7.5 \\
\hline Pasco & 507 & 117 & 23.1 \\
\hline Piura & 6026 & 3111 & 51.6 \\
\hline Puno & 2758 & 430 & 15.6 \\
\hline San Martín & 1832 & 1273 & 69.5 \\
\hline Tacna & 1795 & 238 & 13.2 \\
\hline Tumbes & 580 & 296 & 51 \\
\hline Ucayali & 2004 & 1438 & 71.8 \\
\hline
\end{tabular}

1/ Incluye vehículos menores como moto y motocar

Fuente: INEI. Encuesta de comisarías sobre accidentes de tránsito 2009 y 2010 


\section{Figura 1}

Perú: Accidentes de tránsito, según departamento, 2016

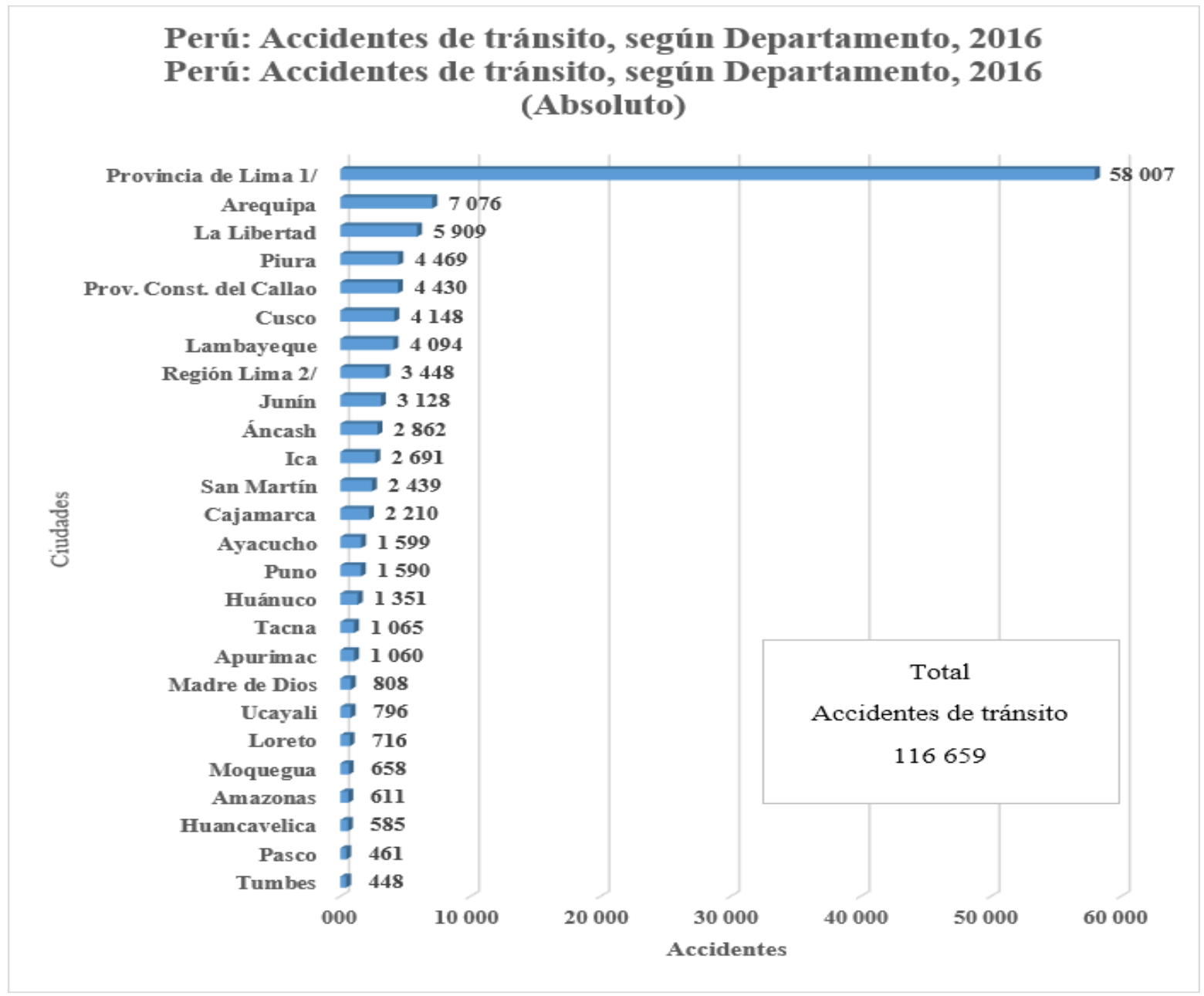

1/ Comprende 43 distritos de la provincia de Lima

2/ Se refiere a la región Lima que comprende las provincias de Barranca, Cajatambo, Canta, Cañete, Huaral, Huarochirí, Huaura, Oyón y Yauyos

Fuente: INEI - VI Censo Nacional de Comisarías 2017

Los datos indicados del año 2009 y 2010 son referenciales vs. los datos actuales que tiene el INEI, son del año 2016, como se muestra en la Figura 1. Es evidente que la bicicleta hoy en día se ha convertido en el vehículo con mayor incremento de compra para su utilización, y son pocas las empresas que han implementado los estacionamientos para bicicletas, a pesar de que todavía se labora de manera virtual; sin embargo, han incrementado el servicio de distribución por ser el medio más económico. 


\section{Figura 2}

Perú: Vehículos menores involucrados en accidentes de tránsito, según tipo de vehículo, 2016

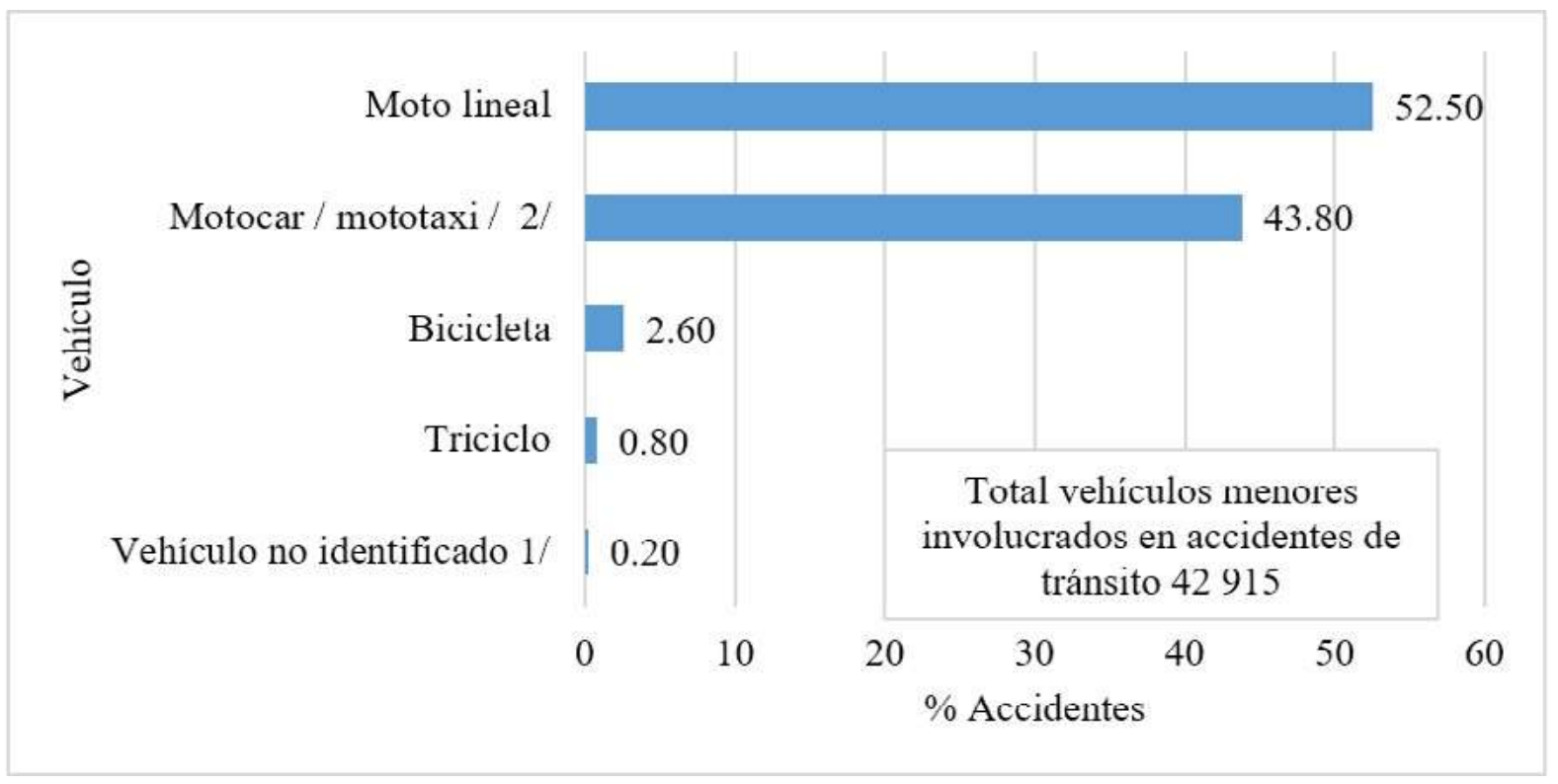

En la Figura 2, el INEI, muestra que el $2.60 \%$ de los accidentes en vehículos menores, corresponde a que una bicicleta estuvo involucrada, y esto se debe, básicamente en que los ciclistas no observan los lineamientos de tránsito y a estos no los respetan los conductores de vehículos mayores, como autos, camionetas, camiones y otros, son datos que corresponden al año 2016; sin embargo, es necesario dar un vistazo a las cifras no oficializadas por el INEI, pero que aparecen en otros medios de comunicación masiva, por lo que podemos asegurar que con la pandemia del SARS-CoV-2, la bicicleta es el vehículo que ha logrado ser tendencia, y algunas de las empresas alientan su uso, pero falta más, y solo se podrá lograr este objetivo con los planes de desarrollo urbano donde estén involucradas las ciclovías, incluso, como ya se ha indicado, son motivo de tesis, pues la generación de los millennial son los más adeptos a movilizarse mediante este medio.

Por otro lado, es también importante que exista infraestructura adecuada, y que tanto el gobierno local como las empresas, sea cual fuere su tamaño, deben de promover el uso de estos vehículos menores, y que para su tránsito se hace necesaria la construcción de más ciclovías en Lima y la implementación de ellas en otras ciudades del país.

En lo que va del año 2021, de enero a abril, van 15 accidentes fatales en bicicleta, casi el doble que en el 2019, y esto se da porque los ciclistas desconocen el reglamento de tránsito, mientras que en el año 2019 solo fueron 8 (El Peruano, 2020).

Llama la atención que, a pesar de la cuarentena, los vehículos que no debieran salir circulan con normalidad y no respetan las señales de tránsito o las ciclovías, y que estas no tengan la seguridad determinada. Por ello, se necesita una solución integral en la que se unan la empresa privada, el gobierno local y demás interesados para que no se vean accidentes de tránsito "masivos" con la consecuente muerte de la víctima.

La bicicleta es ahora, un vehículo de uso masivo, no solo para mejorar la salud, sino porque es el vehículo permitido en época de pandemia (SARS-CoV-2), y porque marca "tendencia", de ahí, también, que los accidentes de tránsito disminuyeron un $70 \%$ en el Perú, dato obtenido hasta diciembre 2 del 2020 (CTC Callao, 2020). 
Además, podemos ver que dentro del ámbito estructural no se cuenta con rutas definidas que brinden una adecuada circulación y mucho menos el aprovechamiento de estas vías, debido a que no están unidas unas con otras. Esto es una desventaja que reduce el uso de estas vías como ruta alternativa a los vehículos motorizados, dificultad principal de la Municipalidad Metropolitana de Lima, ya que es la encargada de velar por el implemento y mantenimiento de estas vías. Además, en el año 2019, ascendió la pobreza al $20.2 \%$, de acuerdo al INEI, mientras que hasta el 19 de febrero del 2020, el nivel de pobreza en Perú llegó al $20.5 \%$, lo cual significa más de 6,4 millones de peruanos, población que se encuentra habida de transporte; asimismo, constituir empresas en las que su principal herramienta de trabajo sea este vehículo sin motor, no contaminante, además de evitar cansancio a diferencia de los vehículos motorizados que se abona el aburrimiento y reduce la productividad de las personas (Ponce de León 2012, 5). Esto demuestra que, según los datos estadísticos, el uso de los vehículos no motorizados (bicicletas) como medio de transporte alternativo sería una actitud factible para el desarrollo de un nuevo medio de transporte, una alternativa para la disminución y el ordenamiento de la ciudad. Esto se puede observar, ya que el empleo de este medio de transporte no consume combustible, no contamina el medio ambiente y sobre todo actúa como regulador de tránsito de las vías públicas.

Con todo esto, conseguir un automóvil es más comercial que obtener una bicicleta, pese que esta última tenga un menor costo monetario. De esto se puede desligar que existe una precaria información sobre el uso de vías alternas para mejorar la calidad de vida y evitar el tráfico en hora punta.

Podemos observar también que el desorden provocado en las vías de tránsito es causado en su totalidad por vehículos motorizados. Asimismo, debemos indicar que, de acuerdo con el reporte de la Policía de Tránsito, en Perú, en el año 2019, hubo 15 accidentes fatales en los que incluía una bicicleta. Esto, en definitiva, demuestra el incremento del uso de la bicicleta como medio de transporte; sin embargo, el poco respeto que se le tiene al ciclista y el desconocimiento de las reglas de tránsito, es la causa que se produzcan estos accidentes, y seguramente irá in crescendo en la medida que los choferes de los vehículos motorizados no observen las señales de tránsito (Agencia AFP, 2020).

Además, otro punto muy importante es la locomoción pública, que en la actualidad tiene una antigüedad entre 15 a 20 años y, por tanto, un mantenimiento limitado en cuanto a repuestos se refiere. Este factor, aunado a la falta de capacitación de los choferes que acceden a una licencia A2 y A3, hacen que el tránsito sea caótico. Sin embargo, se hace masiva la constitución de empresas destinadas al transporte público, en la que se afilian los vehículos con permisos del Ministerio de Transporte y Comunicaciones, sin prever el peligro que representan los choferes para con la sociedad, al no respetar a los vehículos menores ni a los peatones. Esto conlleva a una irregularidad en las empresas que a su vez generan diversas faltas para formalizarse dentro del transporte público. Además, demuestra que las empresas de transporte público no cumplen con ciertas normas, lo cual puede generar un maltrato a los trabajadores debido a sus largas horas de trabajo, lo cual culmina en una mala atención por parte de los cobradores a los pasajeros.

\section{RESULTADOS}

Tacna está ubicada en la costa sur occidental del Perú, sus coordenadas geográficas se sitúan entre $16^{\circ} 58^{\prime}$ y $18^{\circ} 20^{\prime}$ de latitud sur, y $69^{\circ} 28^{\prime}$ y $71^{\circ} 02^{\prime}$ de longitud oeste, por muchos, considerada como una ciudad ubicada estratégicamente, por razones geopolíticas, pues al ser zona de frontera, no solo tiene afluencia extranjera, sino que también existe un importante turismo interno. Con una población de 346 000 habitantes, con un nivel de confianza de $95 \%$ y un margen de error del $3 \%$, el tamaño de la muestra es de 1065 personas, para efectos de conocer lo que piensan, según sus edades, sobre la posibilidad de 
que las ciclovías se construyan adecuadamente en la ciudad, así como la importancia de que el deporte es bueno para la salud, de los que se han obtenido respuestas interesantes. La encuesta preparada para este artículo fue aplicada entre el 13 a 23 de julio del 2021 y lanzada a través de las redes sociales y por QuestionPro y formularios de Google solo en la ciudad de Tacna, arrojaron los siguientes resultados:

\section{Figura 3}

La edad referente en la que manejan un ciclo

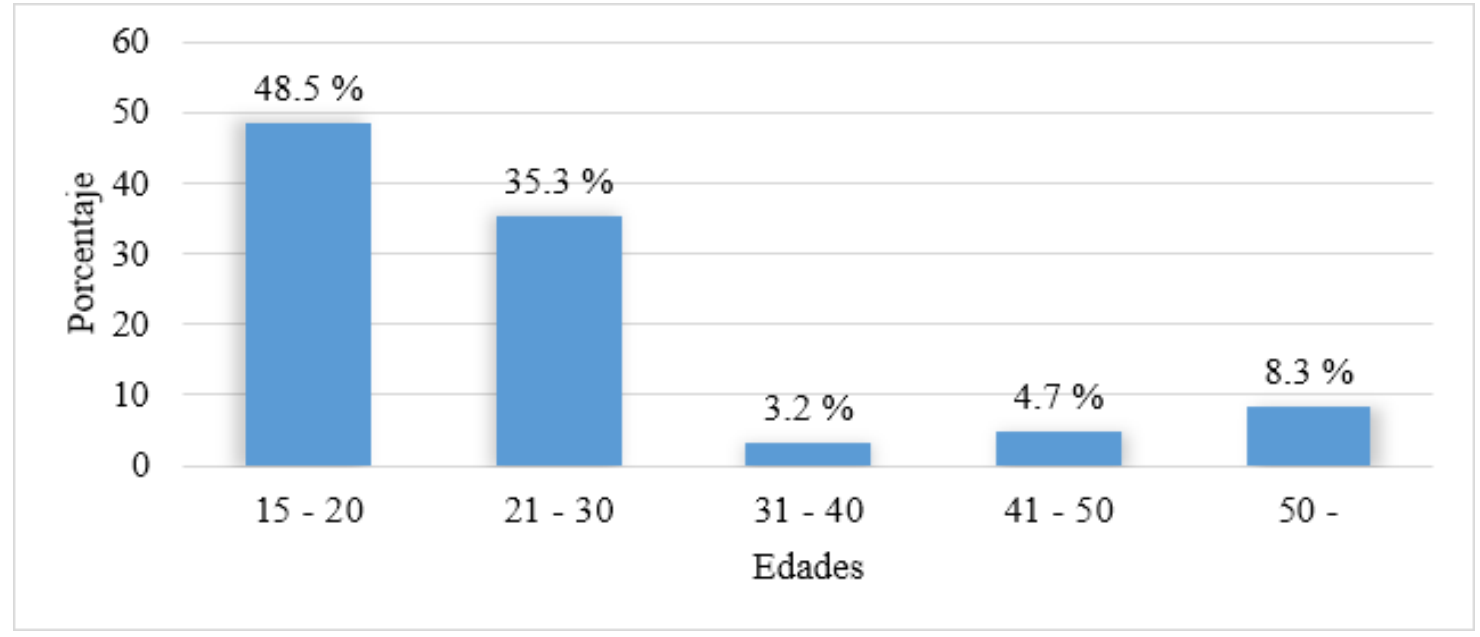

En esta figura podemos verificar que la más alta población conformada por las edades de 15 a 20 años representa el $48.5 \%$ que maneja un ciclo, mientras que el $35.3 \%$ que significa la población entre 21 a 30 años, el $8.3 \%$ refiere a los mayores a 50 años, entre 41 a 50 años traducidos en el $4.7 \%$, y el porcentaje más bajo, equivalente al $3.2 \%$, son los del tramo de 31 a 40 años. Por tanto, la población que más utiliza un ciclo se encuentra entre los 15 a 30 años significa el $83.8 \%$.

\section{Figura 4}

¿Hace ejercicio?

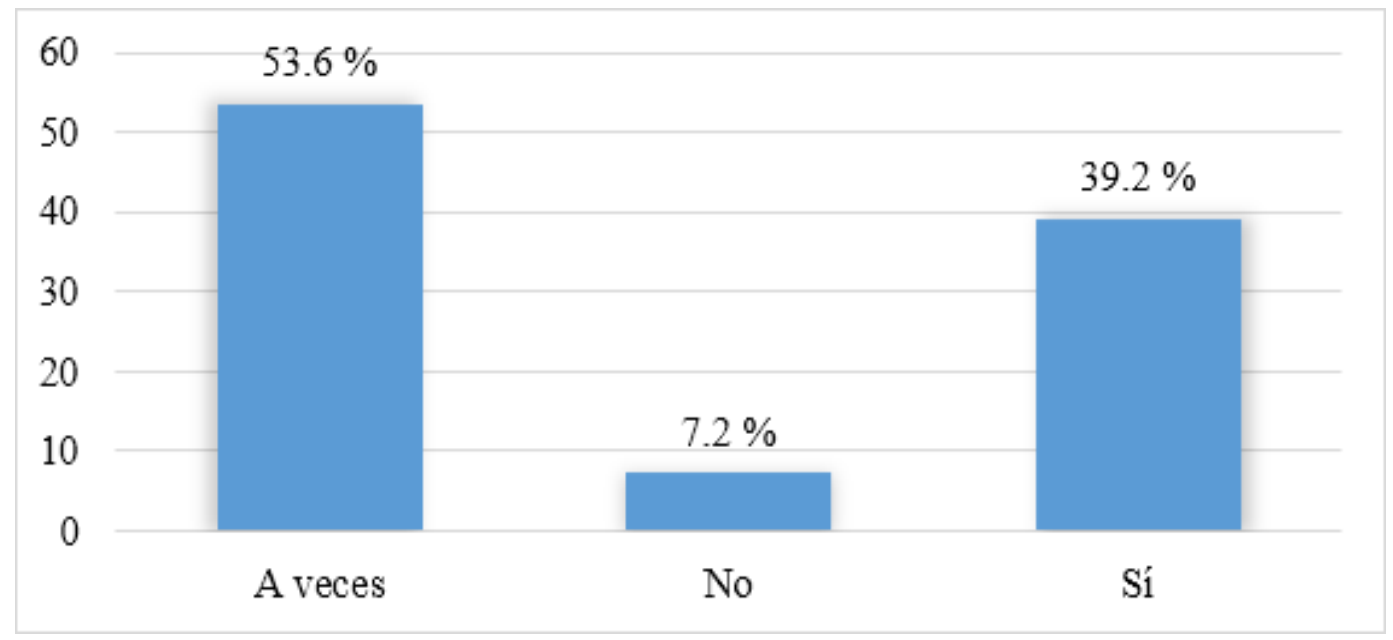

Esta figura muestra que solo el $39.2 \%$ practica ejercicio, mientras que el $53.6 \%$ solo lo hace a veces, y el $7.2 \%$ no hace ejercicio, por tanto, no utiliza ningún ciclo. 


\section{Figura 5}

¿Qué tipo de vehículo tiene?

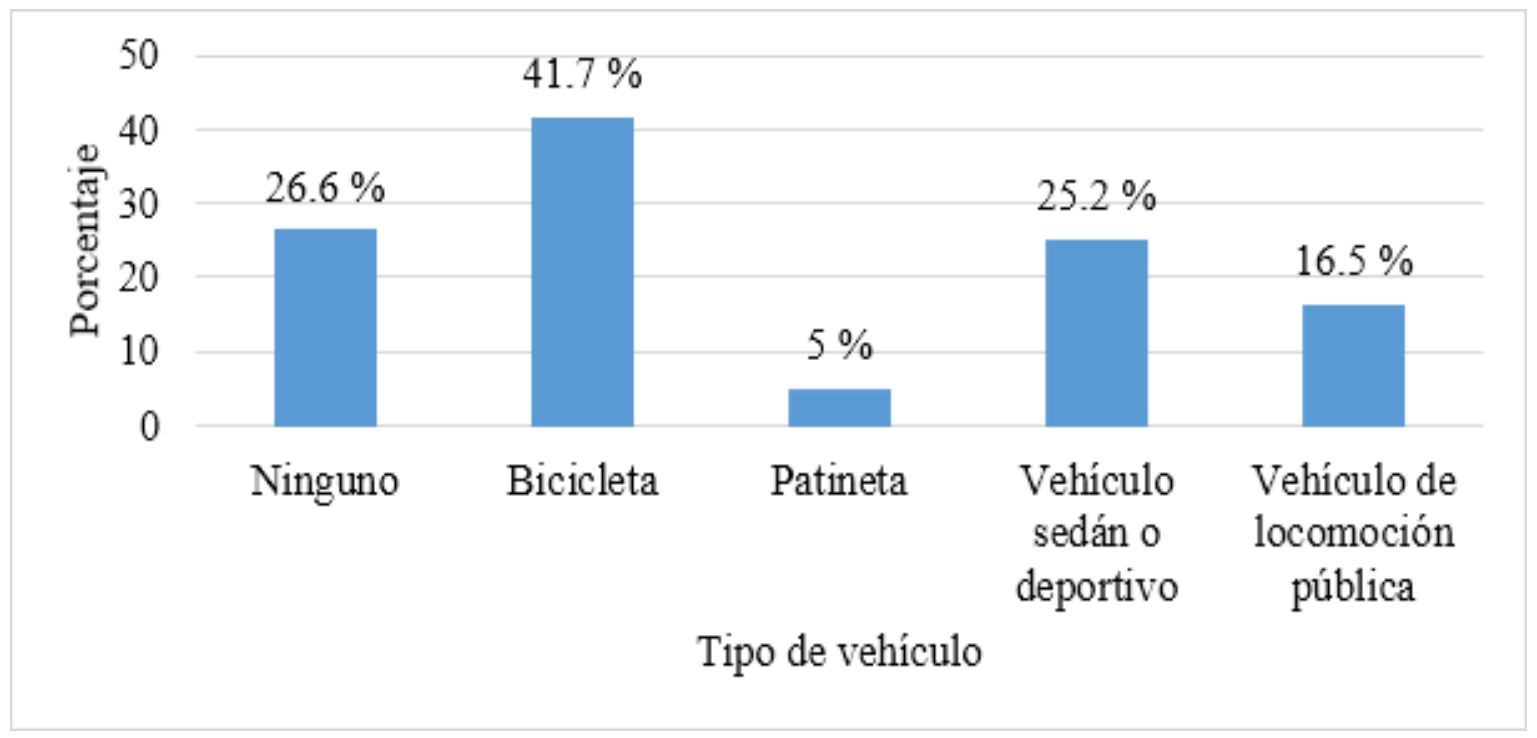

Con las Figuras 5 y 6, se ratifica la Figura 4, pues se tiene que entre el 59.7 al $68 \%$ no tiene o maneja un ciclo, lo que quiere decir que no hace ejercicio de este tipo.

\section{Figura 6}

¿Qué tipo de vehículo maneja?

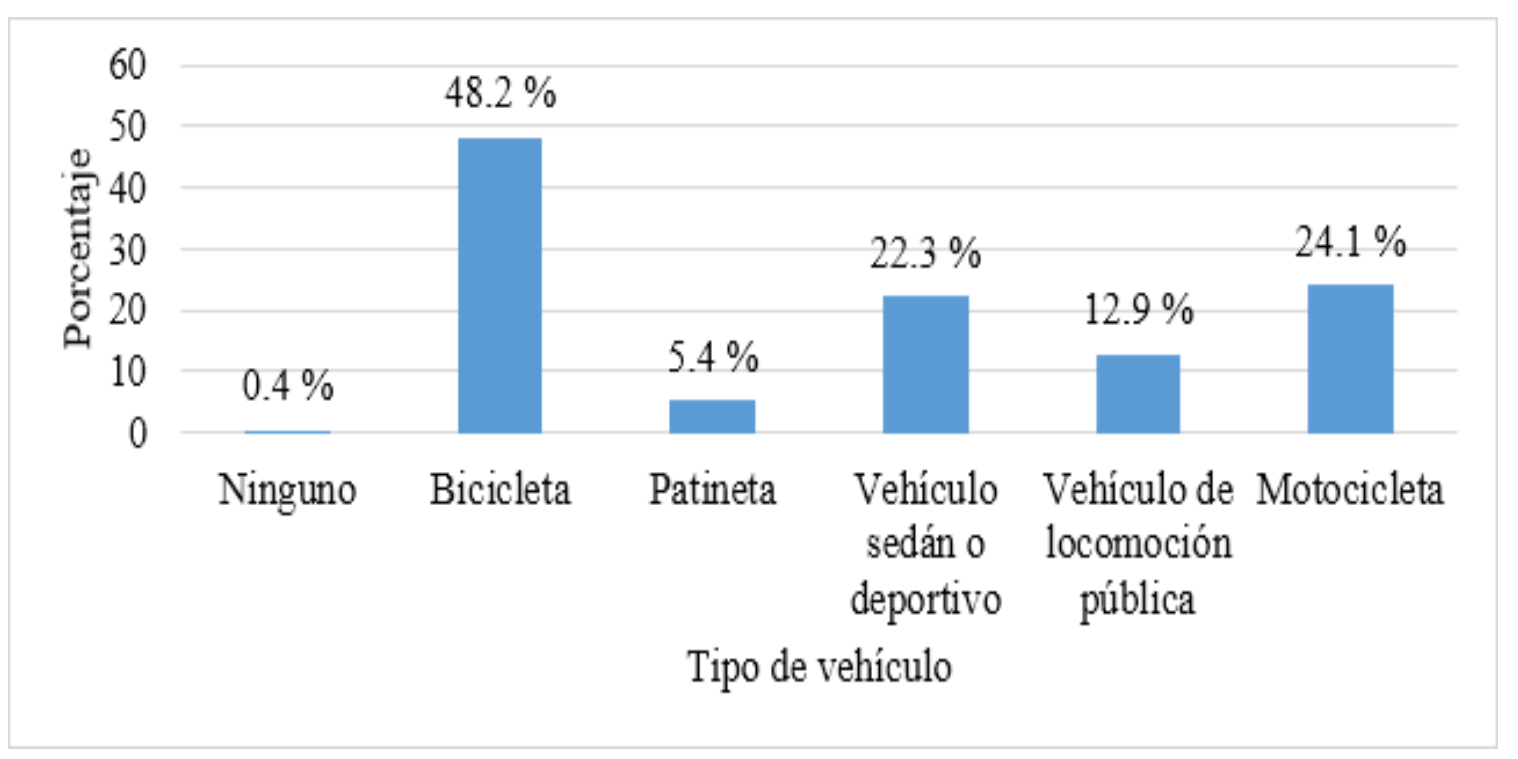

A través de la Figura 6, se indica que un $48.2 \%$ maneja bicicleta y el $5.4 \%$ patineta, lo cual da $53.6 \%$, porcentaje que contrasta con la Figura 4 en la que se determina que el $53.6 \%$ solo realiza ejercicio a veces y el $7.2 \%$ ninguno. 


\section{Figura 7}

¿Qué tan importante cree usted que es el ejercicio para la salud?

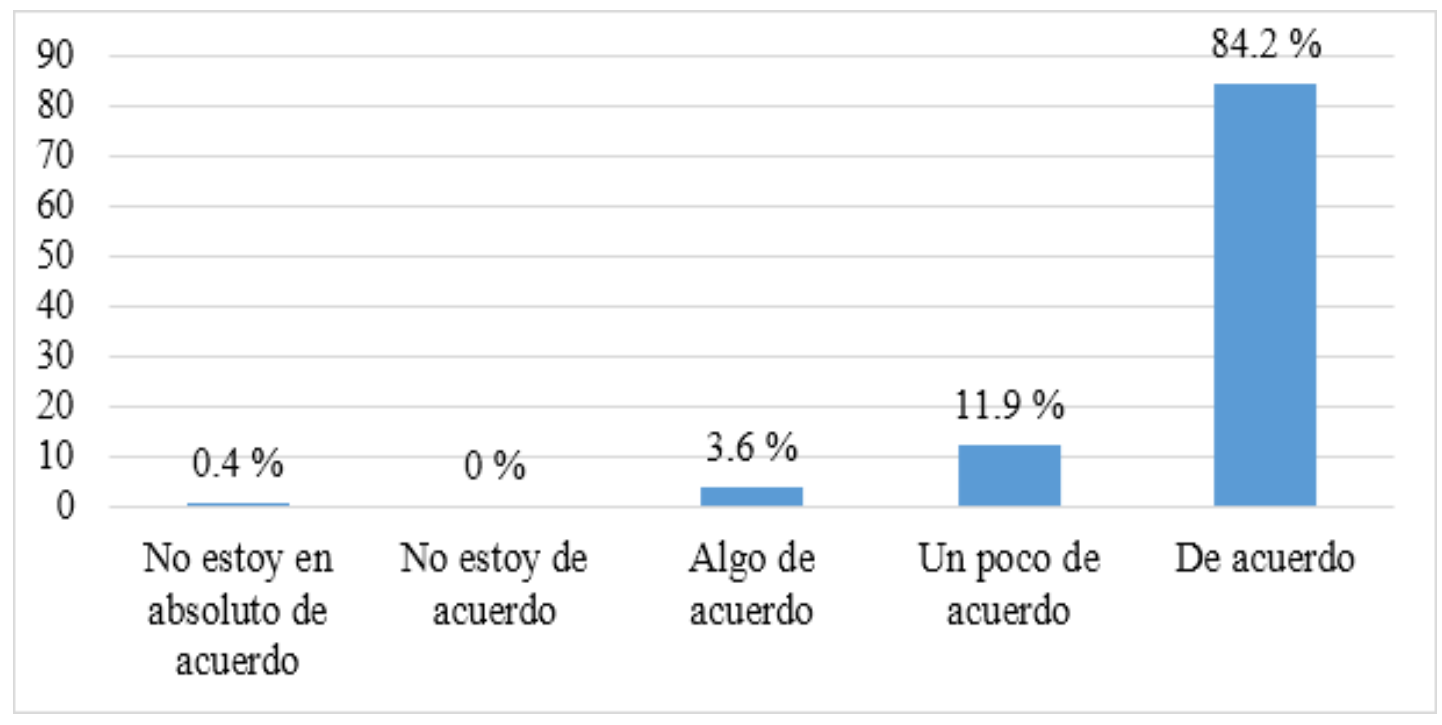

Sin embargo, en la Figura 7, se muestra que el $84.2 \%$ cree que el ejercicio es bueno para la salud, lo que contradice el actuar de la población encuestada, que, a pesar de estar conscientes de la importancia del deporte, lo practica a veces o no lo practica (Figura 4). El $11.9 \%$ está un poco de acuerdo, mientras que $3.6 \%$ está algo de acuerdo, e increíblemente, aunque diminuto el porcentaje de $0.4 \%$ no está de acuerdo.

\section{Figura 8}

Para movilizarse dentro de la ciudad de Tacna, ¿qué utiliza?

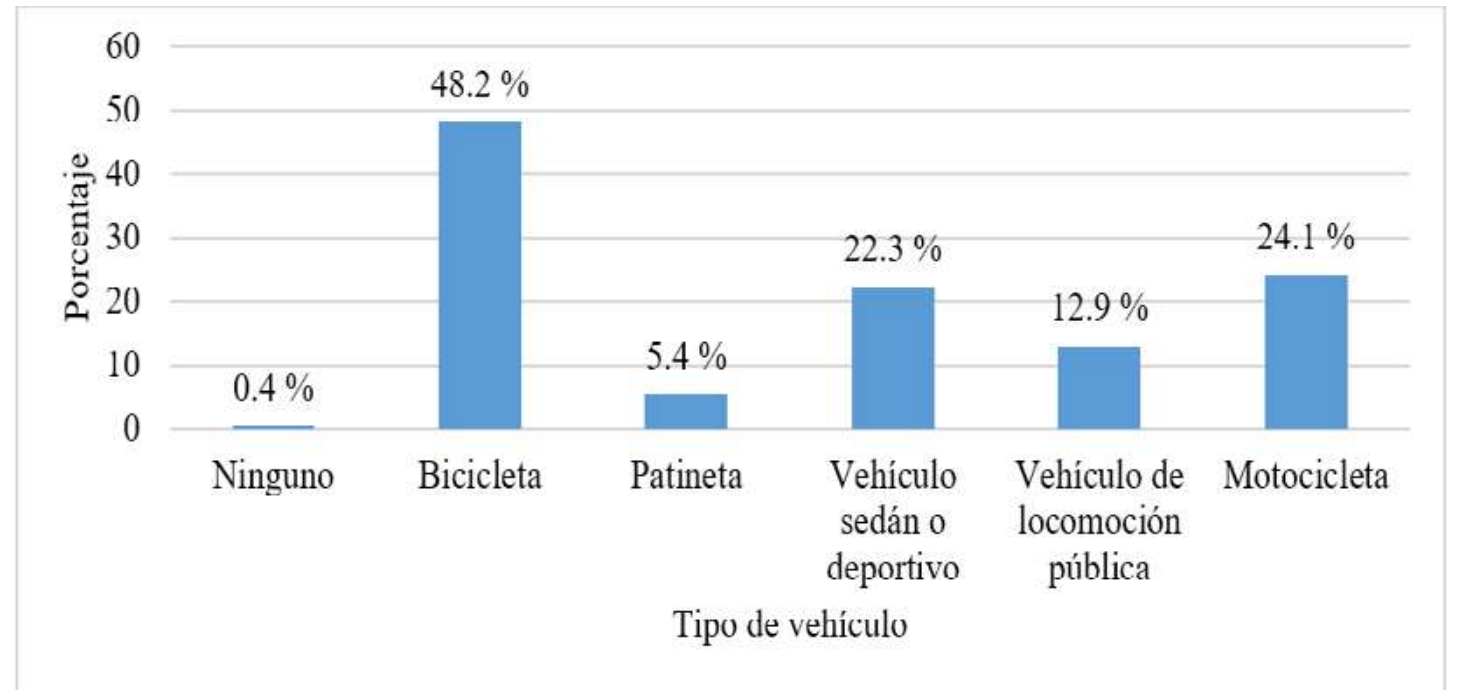

En la figura antecedente, se muestra que, para movilizarse, la bicicleta es utilizada en $48.2 \%$, la patineta en $5.2 \%$, y el $0.4 \%$ ninguno; es decir, que caminan, mientras el $22.3 \%$ utiliza vehículo sedán o deportivo, y el $12.9 \%$ utiliza vehículo de locomoción pública. 


\section{Figura 9}

¿Cree que la infraestructura de la ciudad de Tacna es la adecuada para construir ciclovías?

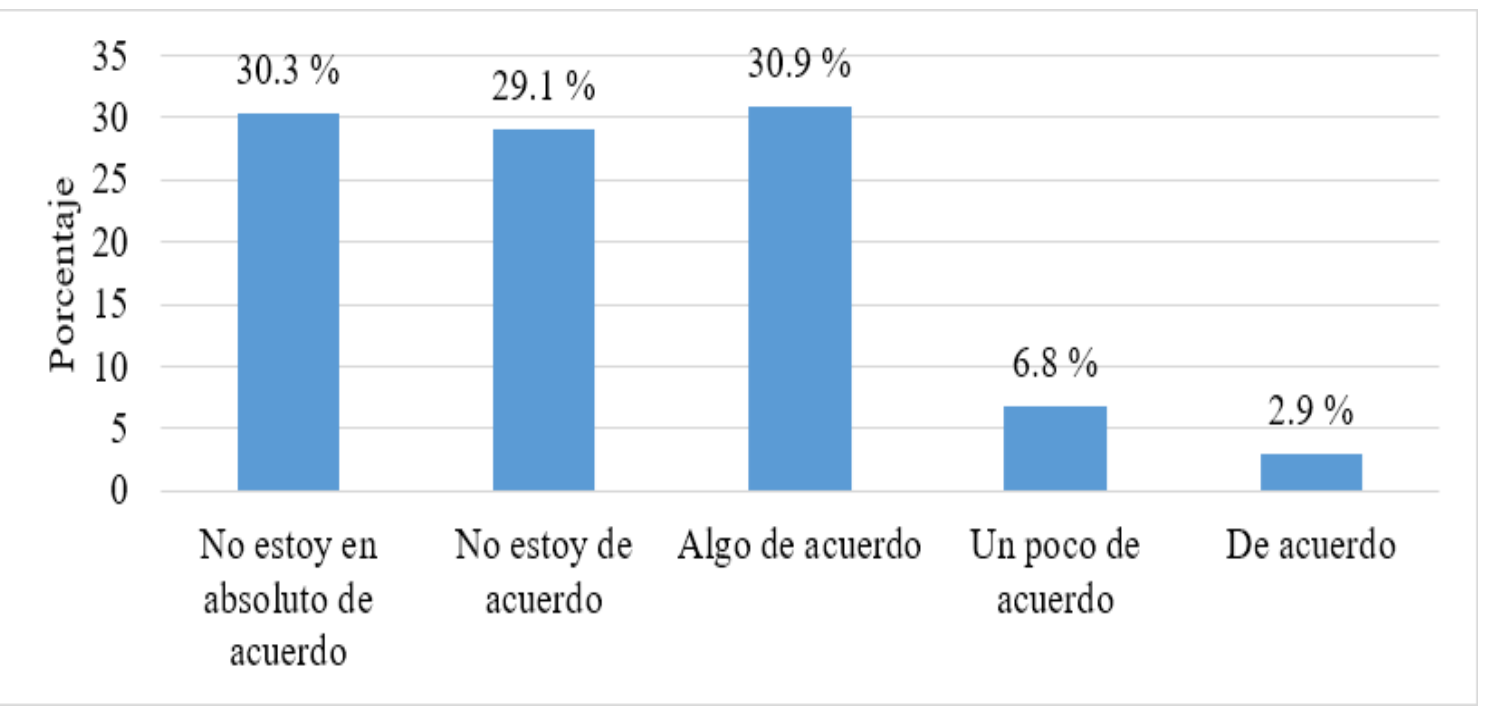

En la Figura 9, se muestra que un importante $30.3 \%$ no está en lo absoluto de acuerdo en que la infraestructura de Tacna sea la adecuada para la construcción de ciclovías, mientras que el $30.9 \%$ está algo de acuerdo, y el $29.1 \%$ no está de acuerdo. Esto se debe a la estrechez de las calles y avenidas existentes en la ciudad, por tanto, solo el $6.8 \%$ está un poco de acuerdo y el $2.9 \%$ de acuerdo.

\section{Figura 10}

¿Quién debe desarrollar o fortalecer las ciclovías en el Perú?

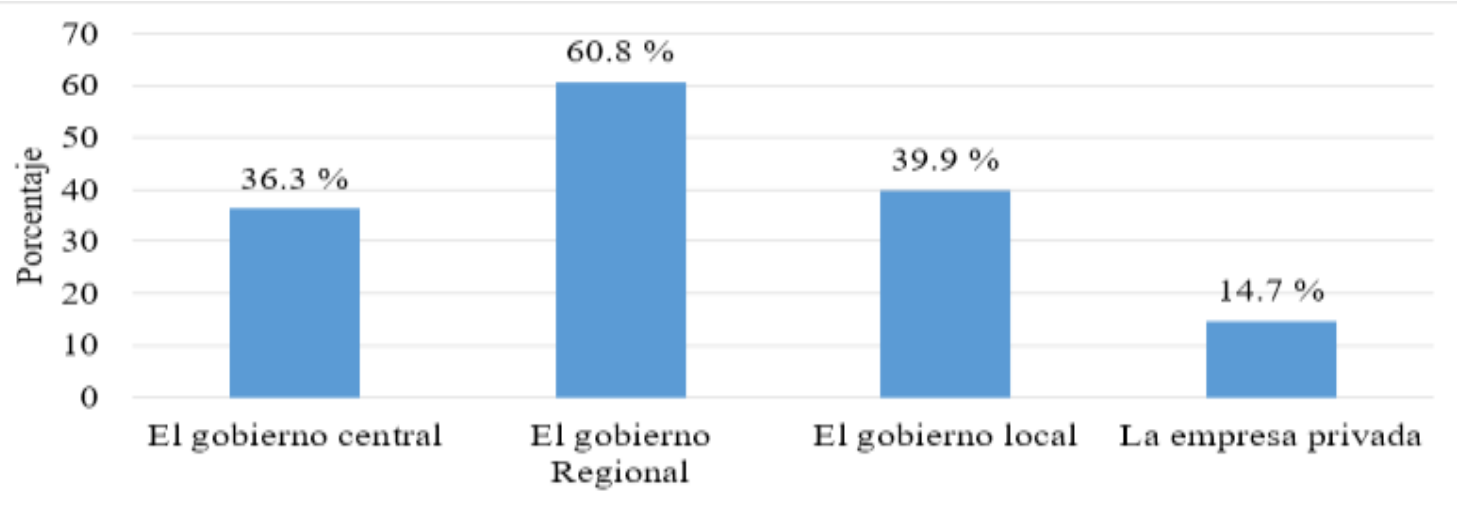

Finalmente, tenemos que a pesar de que la legislación ya se dio y se tiene el presupuesto que asignar, de acuerdo con los proyectos de cada región y gobierno local, el $14.7 \%$ piensa que es la empresa privada la que debe desarrollar o fortalecer las ciclovías en el Perú, mientras que el $39.9 \%$ piensa que sería de responsabilidad del gobierno local, un importante $60.8 \%$ piensa que el gobierno regional debe ser el responsable, y el $36.3 \%$ el gobierno central.

\section{DISCUSIÓN}

En la actualidad, en Tacna, se están recopilando firmas para presentar un proyecto que uniría, a través de ciclovías, los distritos de Tacna, Ciudad Nueva, Alto de la Alianza y Pocollay. Sin embargo, en Tacna cuadrada, se hace dificultosa o poco probable su construcción por el ancho de las pistas urbanas, ya que en caso de emergencias los vehículos no pueden fluir en doble sentido. 


\section{Resultados del estudio empírico}

Ha quedado demostrado que el uso de la bicicleta como deporte y transporte necesario, es el más adecuado con el objetivo de lograr una mejor salud, armonía en los trabajos, el hacer sentir mejor el organismo, como una forma de confraternizar con los compañeros de trabajo, mejorar el clima organizacional y el comportamiento, toda vez que los colaboradores llegarán desestresados, con ánimo de trabajar, precisamente por la endorfina, serotonina, dopamina y oxitocina que se segregan cuando se hace ejercicio.

El ideal es que el colaborador tenga sus objetivos alineados a los de la empresa y que sus aspiraciones puedan ser satisfechas por ella, de ahí que se hace importante que las empresas, dentro de su responsabilidad empresarial, contemplen que sus colaboradores se movilicen en bicicleta, o hacer eventos en los que se utilice este importante vehículo que no contamina y que por el contrario, los beneficios son varios, además de abrirse varias ideas de emprendimientos que van desde la compraventa de bicicletas, su innovación, como las hechas con cañas de bambú, y la producción y venta de accesorios para quienes las manejan, otorgándose la seguridad correspondiente y de acuerdo a la nueva normatividad ya indicada.

De la misma forma, queda demostrado que, en Tacna cabe la posibilidad de construir infraestructura adecuada para ciclos, según los espacios y lugares, con rutas específicas, lo que se traduciría esencialmente en ciclovías para deporte y transporte a lugares limitados, como las pistas que van hacia los distritos de Tacna: Alto de la Alianza, Ciudad Nueva, Pocollay, Calana, Gregorio Albarracín, Inclán, La Yarada Los Palos, Pachía, Pocollay, Sama y CPM Boca del Río.

En la ciudad de Tacna, las calles son angostas e imposibles de anchar, más aún se debe considerar los casos de emergencia, por lo que hay avenidas en las que no es posible señalizar los espacios de ciclovías con palitroques 2 , pues las ambulancias y vehículos de bomberos u otros no podrían tener una rápida circulación, ya que los vehículos que transitarían por esas calles o avenidas no tendrían espacio para colocarse a un lado en caso de que el vehículo de emergencia requiera pasar, no habría fluidez y seguridad.

\section{CONCLUSIONES}

Las vías de transporte alternativas brindan una mejora que podría visualizarse en el día a día mediante la disminución del tráfico vehicular en Lima Metropolitana. En conjunto con las normas, vías de tránsito y una implementación de infraestructura se puede llegar a consolidar al uso de ciclovías como una forma viable, económica y promedio ambientalista que mejoraría la calidad de vida dentro de la ciudad. Con el implemento de esta vía de transporte se mejorarían dos partes importantes en la sociedad limeña: (i) Una disminución de vehículos motorizados; (ii) Mejora en la calidad de vida; (iii) Se evitaría el tráfico dentro de las principales avenidas y calles, lo cual permitiría que se usen vías alternas para un mejor aprovechamiento de las ciclovías.

Podemos indicar que, tanto las empresas privadas como estatales, deben invertir un presupuesto que permita la restauración y el mantenimiento en óptimas condiciones de las vías. Por otro lado, las soluciones que plantean algunas empresas sobre el uso de las apps (Applications) resultan una solución con la cual se pueda llegar a este fin. Sin lugar a duda, se puede precisar que el principal impedimento en transitar en las ciclovías es la falta de rutas y conexión entre las diferentes ciclovías de los distritos. Es por eso por lo que se ha realizado un plan para crear unas vías más seguras y regidas por normas para que se pueda ver un orden al momento de hacer el uso de estas vías.

$\overline{\text { 2Palo pequeño }}$ 
Es de gran importancia la normatividad publicada en el año 2020, referente a la importación de vehículos usados, así como la reglamentación del uso de bicicletas, de forma que promueve e impulsa la construcción de ciclovías, lo que hará que el auge del uso de este vehículo se incremente, lo que contribuye al objetivo de lograr una vida más sana de la población.

En consecuencia, en Tacna, como en el resto del país, se hará más fácil la construcción de infraestructura adecuada para las ciclovías, en la medida que ya existe la normatividad pertinente para el financiamiento de las pistas.

En Tacna, la habilitación de ciclovías es más probable implementarlas en las pistas que van hacia los distritos, en la medida que son anchas y de doble vía.

La gran mayoría de la población de Tacna afirma que el ejercicio es importante para la salud; sin embargo, son pocas las veces que se ejercitan, por lo que en contexto de las figuras que se han señalado en este trabajo, concluimos que los ciclos son utilizados, y estos se incrementarían si se habilitan ciclovías adecuadas.

Entonces, podemos finalizar haciéndonos la siguiente pregunta. ¿Es viable que en Lima Metropolitana y en otras ciudades, entre ellas Tacna, se pueda implementar el uso de las ciclovías como medio de transporte alternativo? Esta cuestión ayuda a una mejor interpretación de los errores cometidos, tanto por el lado de la Municipalidad Metropolitana de Lima como por parte de los usuarios, y así poder explicar que el uso de este tipo de medio de transporte puede conllevar a cambios beneficiosos en la sociedad limeña viéndose beneficiada por la eficiencia del medio, la mejora de la salud física y mental y sobre todo un medio ambiente libre de contaminación, sin dejar de lado el fin de una Lima desordenada y caótica por el incremento vehicular, lo que implica no solo la viabilidad de la implementación del uso de las ciclovías, sino que también, se demuestra su necesidad. Prueba de ello, son algunos estudios e investigaciones efectuadas por estudiantes de la PUCP, que incluso, han sido materia de tesis que responden a esta pregunta como: "Uso de bicicletas como transporte urbano seguro. Caso Surco" o "Plan maestro de ciclovías para el área metropolitana de Lima y Callao", entre otras. Por último, el cambio a este nuevo sistema representaría el cambio a una nueva ciudad ordenada.

\section{REFERENCIAS}

Ponce de León, Oscar (2012) Los retos del desarrollo de infraestructura de transporte urbano en Lima Metropolitana a través de asociaciones público-privadas (consulta 8 de noviembre del 2014) http://cies.org.pe/sites/default/files/investigaciones/los-retos-del-desarrollo-deinfraestructura-de-transporte-urbano-en-lima.pdf

Instituto Nacional de Estadística e Informática (2017). Análisis de los Accidentes de Tránsitos Ocurridos en el año 2016. Instituto Nacional de Estadística e Informática, Lima, Lima. Recuperado el 22 de abril de 2021, de https://www.inei.gob.pe/media/MenuRecursivo/publicaciones_digitales/ Est/Lib1528/cap03.pdf

Instituto Nacional de Estadística e Informática (2014) Misión y Visión (consulta: 8 de noviembre del 2014) (http://www.inei.gob.pe/mision-y-vision/)

Agencia AFP (28 de diciembre de 2020). Muertes de ciclistas en Perú casi se duplicaron en el 2020 por la pandemia. Gestión, pág. 2/5. Recuperado el 14 de abril de 2021, de https://gestion. pe/peru/muertes-de-ciclistas-en-peru-casi-se-duplicaron-en-el-2020-por-la-pandemianoticia/?ref=gesr

El Peruano (27 de diciembre de 2020). El Peruano. Recuperado el 16 de abril de 2021, de El Peruano: https://elperuano.pe/noticia/112491-este-ano-van-15-accidentes-fatales-en-bicicleta-casi-eldoble-que-en-2019 
Perú, E. (Ed.). (03 de junio de 2020). Decreto Supremo que aprueba el Reglamento de la Ley $\mathrm{N}^{\circ}$ 30936, Ley que promueve y regula el uso de la bicicleta como medio de transporte sostenible, modifica el Reglamento Nacional de Tránsito, aprobado por Decreto Supremo No 033-2001MTC. Decreto Supremo Nº12 - 2020 - MTC (15461), 56 (1-17). Lima, Lima, Perú: Empresa Peruana de Servicios Editoriales S.A. Recuperado el 10 de mayo de 2021, de https://busquedas. elperuano.pe/download/url/decreto-supremo-que-aprueba-el-reglamento-de-la-ley-n-30936decreto-supremo-no-012-2020-mtc-1867296-1

Perú, E. (Ed.). (02 de febrero de 2020). El Peruano. Decreto Supremo No 005-2020-MTC (15261), 88 (30-31). Lima, Lima, Perú: Empresa Peruana de Servicios Editoriales S.A. Recuperado el 10 de mayo de 2021, de https://busquedas.elperuano.pe/download/url/modifican-los-requisitosminimos-de-calidad-para-la-importac-decreto-supremo-n-005-2020-mtc-1851548-7

Perú, E. (Ed.). (03 de junio de 2020). Decreto Supremo que aprueba el Reglamento de la Ley $\mathrm{N}^{\circ}$ 30936, Ley que promueve y regula el uso de la bicicleta como medio de transporte sostenible, modifica el Reglamento Nacional de Tránsito, aprobado por Decreto Supremo N ${ }^{\circ}$ 033-2001MTC. Decreto Supremo No 012 - 2020 - MTC (15461), 56 (1-17). Lima, Lima, Perú: Empresa Peruana de Servicios Editoriales S.A. Recuperado el 10 de mayo de 2021, de https://busquedas. elperuano.pe/download/url/decreto-supremo-que-aprueba-el-reglamento-de-la-ley-n-30936decreto-supremo-no-012-2020-mtc-1867296-1

Municipalidad de Lima (2013) Manual de implementación y promoción de Ciclovías Recreativas (consulta 8 de noviembre del 2014) http://www.bvsde.paho.org/documentosdigitales/bvsde/ texcom/cd045364/ciclovias.pdf

Castro, Alicia (2005) Políticas Municipales para la Promoción de la Actividad Física (consulta 8 de noviembre del 2014) https://www.mtc.gob.pe/portal/consultas/cid/Boletines_CID/16_ NOVIEMBRE/ARCHIVO/politicas.pdf

CTC Callao. (2 de diciembre de 2020). Consorcio Tránsito Ciudadano. Recuperado el 15 de abril de 2021, de CTC Callao: http://www.ctccallao.com.pe/los-accidentes-de-transito-disminuyeronun-70-en-el-peru-por-la-pandemia-del-coronavirus/\#: :text=(01)\%20\%2D\%206147373,Los\%20accidentes\%20de\%20tr\%C3\%A1 nsito\%20disminuyeron\%20un\%2070\%25\%20 en\%20el,por\%20la\%20pandemia\%20del\%20Cor

Defensoría del pueblo (2008) El transporte Urbano en Lima Metropolitana: Un desafío en defensa de la vida (consulta 8 de noviembre del 2014) (http://ditoe.minedu.gob.pe/Materiales\%20DITOE/ B14.pdf)

Lao, R. T. (27 de junio de 2021). gob.pe. Recuperado el 13 de julio de 2021, de gob.pe: https://www. gob.pe/institucion/minsa/noticias/43091-menos-del-12-de-peruanos-realiza-actividad-fisicaexclusiva-para-beneficio-de-la-salud

Ministerio de Transportes y Comunicaciones. (22 de setiembre de 2020). Manual para ciclistas del Perú - Reglas y recomendaciones para el uso de la bicicleta y otros ciclos. Recuperado el 25 de julio de 2021, de Manual para ciclistas del Perú: https://cdn.www.gob.pe/uploads/document/ file/1309978/Manual\%20para\%20ciclistas\%20del\%20Per\%C3\%BA.pdf

Gordon Prieto Ghislaine, A. J. (2019). Universidad del Pacífico - Repositorio Institucional. Plan de negocio: cycle tours vive la experiencia: tours E-Bike y E-Scooters. Lima, Lima, Perú. Recuperado el 15 de abril de 2021, de http://hdl.handle.net/11354/2695

Herrera, J. M. (2017). Proyecto inversión pública ciclovías y calidad de vida del adulto mayor Municipalidad de Surco-Lima-2016. Lima, Lima, Perú. Recuperado el 14 de abril de 2021, de 
https://repositorio.ucv.edu.pe/handle/20.500.12692/6805

Landa, M. C. (01 de 09 de 2014). PUCP. Análisis del diseño de la política de la calidad del aire de la Municipalidad Metropolitana de Lima 2010 - 2012. Lima, Lima, Perú. Recuperado el 14 de abril de 2021, de PUCP: http://tesis.pucp.edu.pe/repositorio/handle/20.500.12404/5553

Mogrovejo Jaramillo, P. E. (02 de febrero de 2016). Determinación de los factores de riesgo cardiovascular y comportamientos para la práctica de actividad física y el consumo de frutas y verduras en trabajadores de una empresa privada Ecuatoriana en el 2006. Catalunya, Barcelona, España. Recuperado el 13 de julio de 2021, de http://hdl.handle.net/2117/96260

Vila, J. A. (17 de diciembre de 2015). El uso de la bicicleta como alternativa de transporte sostenible e inclusivo para Lima Metropolitana: recomendaciones desde un enfoque de movilidad. Lima, Lima, Perú. Recuperado el 14 de abril de 2021, de http://tesis.pucp.edu.pe/repositorio/ handle/20.500.12404/6484

Vilma Tapia, L. C.-V. (03 de julio de 2018). Reordenamiento vehicular y contaminación ambiental por material particulado (2,5 y 10), dióxido de azufre y dióxido de nitrógeno en Lima Metropolitana, Perú. Revista Peruana de Medicina Experimental y Salud Publica, 35(2). doi: http://dx.doi. org/10.17843/rpmesp.2018.352.3250 